\title{
EDITORIAL
}

\section{A special section on semiconductors}

\author{
Mingwang SHAO (ه) \\ Institute of Functional Nano \& Soft Materials (FUNSOM), Jiangsu Key Laboratory for Carbon-Based Functional Materials \& Devices, \\ Soochow University, Suzhou 215123, China
}

(C) Higher Education Press and Springer-Verlag Berlin Heidelberg 2011

Semiconductors are inherently endowed with excellent photoelectric properties. Semiconductor materials are the foundation of modern electronics and many other devices including transistors, solar cells, many kinds of diodes, and digital and analog integrated circuits. Those have changed and will continue having varied impacts on lifestyles of people in many ways by manipulating some powerful new devices and circuits related to semiconductors at atomic, molecular, and nano-scale levels. This special section showcases the exciting achievements on synthesis, characterization, and application of various semiconductors in the effort to provide a broad overview of the frontier of ongoing research in this field. There are 14 original papers in this special section.

P. K. Samanta et al. in India investigated substrate effect on morphology and photoluminescence from $\mathrm{ZnO}$ monopods and bipods. H. X. Wu's group not only proved that large size AgGaGeS4 crystals for infrared conversion could be grown, but also confirmed synthesis and growth of nonlinear infrared crystal material CdSe via seeded oriented temperature gradient solution zoning method. Employing multi-layered Ge/Si nanocrystals for storage fabricated with ALD and PLD methods, Y. Shi's group proved that both the two-layered and three-layered memory structure behaved relatively qualified for the multi-level cell storage, and they also demonstrated that compared to electrons, holes could reach a longer retention time even with an ultra thin tunnel oxide, owing to the high band offset at the valence band between $\mathrm{Ge}$ and Si. Q. Li et al. synthesized flower-like CuS nanostructures in ionic liquid at room temperature and their results indicated that the as-prepared CuS nanostructures are promising candidates in the development of photoelectric devices. D. B. Wang's group completed synthesis of ZnO nano-/micro-rods via solution-based routes and quantitatively analyzed optical property of ZnO nano-/micro-rod. Sliver doped n-type CdS nanoribbons (NRs) were successfully synthesized by J. S. Jie et al. and their optoelectronic properties would greatly favor the application of CdS nanostructures in high-performance nano-optoelectronic devices. L. Zhang et al. observed sensitive surface-enhanced Raman scattering signals of $\mathrm{p}$-aminothiophenol on $\mathrm{Ag}$ nanoparticles and silver molybdate nanowires complex. The work by Y. Y. Shao's group that performed synthesis of silicon nanowires supported Ag nanoparticles and analyzed their excellent catalytic activity in the photo-degradation of Rhodamine B. High-yield synthesized silver orthophosphate nanowires and their application in photoswitch were provided by R. H. Que. Y. H. Tong et al. demonstrated that controllable synthesis of quasi-spherelike $\mathrm{ZnO}$ hierarchical nanostructures and performance of their dye-sensitized solar cells. Z. H. Wang's group obtained trigonal selenium nanowires with uniform sizes and their photoelectrical properties were investigated, which suggested their potential using in the fabrication of micro-devices or photo-switches. Preparation, characterization, and photoconductivity of bis-(8hydroxyquinoline) copper nanoribbons were examined by Y. C. Chen et al. L. Liu and Z. Zhang investigated facile solvothermal synthesis and photoconductivity of one-dimensional organic Cd(II)-Schiff-base nanoribbons.

It is expected that this special section on semiconductors not only presents to the readers an overview about the current ongoing research activities on the international frontier, but also provides the guidance for their future research endeavors and developments. It is difficult to complete this special section without the great supports and efforts of all the authors. Finally, special thanks to Professor Guozhen Shen, the Executive Editor of Frontiers of Optoelectronics in China for his great efforts to help us finish this special section. 